\title{
Cuando los dioses son tiempo. Breve crítica a la temporalidad occidental
}

\author{
Icnitl Ytzamat-ul Contreras García
}

Este texto tiene por objetivo establecer un contraste entre la concepción temporal de la cultura maya-mexica y la concepción temporal cotidiana que tenemos en tanto cultura occidental. El contraste se establecerá en primer lugar a partir de una breve exposición de los calendarios mayas y mexicas, es decir, de la comprensión de los calendarios es que se puede crear un horizonte en el que lo religioso permea toda actividad en ambas culturas. En segundo término exponemos en breves líneas la concepción cotidiana del tiempo, ayudándonos de un filósofo vertebral en el pensamiento moderno occidental: Kant. Al enunciar ambas posiciones buscaremos construir un puente en el que criticaremos la concepción temporal cotidiana de Occidente, ya que ésta tiene sus fundamentos en la ideología que cobró fuerza a partir de la modernidad: el capitalismo y el liberalismo. Para construir dicho puente, nos ayudaremos de un filósofo crítico de la modernidad: Walter Benjamin.

Palabras Clave: Temporalidad, mayas, mexicas, Walter Benjamin.

This text aims to establish a contrast between the temporal concept of MayaMexica culture and the everyday temporal concept of Western culture. This contrast is established first through a brief summary of the Mayan and Aztecs calendars: it is through the understanding of the calendars that one can create a horizon on which religion permeates every activity in both cultures. Secondly, we briefly present the everyday concept of time, relying on a key philosopher in modern Western thought: Kant. By outlining both positions, we seek to build a bridge that will enable us to critique the everyday temporal concept of the West for its foundation on the ideology that gained its strength from moder- 
nity: capitalism and liberalism. To build this bridge, we refer to a critical philosopher of modernity: Walter Benjamin.

Keywords: Temporality, Mayas, Mexicas, Walter Benjamin.

Fecha de recepción: 22 de agosto de 2013

Fecha de aceptación: 7 de noviembre de 2013 
Icnitl Ytzamat-ul Contreras García

Universidad Nacional Autónoma de México

Instituto de Investigaciones Filológicas

\title{
Cuando los dioses son tiempo. Breve crítica a la temporalidad occidental
}

\author{
Sentir todo de todas las maneras, \\ vivir todo de todos los lados, \\ ser la misma cosa de todos los modos posibles al mismo tiempo, \\ realizar en mí toda la humanidad de todos los momentos \\ en un sólo momento difuso, profuso, completo y lejano.
}

Pessoa

El tiempo es un concepto y una problemática que ha sido tratada desde los antiguos filósofos griegos hasta las discusiones contemporáneas en filosofía y ciencia. Platón, precursor fundamental, expuso la conceptualización temporal en el Timeo; no obstante, fue rebasado por Aristóteles, quien abordó la cuestión con mayor profundidad y atención que su maestro en el "Libro Iv" de la Física. Años más tarde, san Agustín trató el tema en el "Libro XI" de las Confesiones, y más recientemente Heidegger, quien lleva el sentido del ser a un punto de encuentro con el tiempo. No profundizaremos en una concepción específica de los estudios filosóficos ni científicos, más bien trataremos de esclarecer cómo comprendemos el tiempo de manera común, cotidiana - tarea por sí misma complicada-, para después someter esa explicación a una crítica a partir de dos elementos: la forma de comprensión temporal maya-mexica, y el cuestionamiento que hace Walter Benjamin del progreso en la modernidad. 
En occidente entendemos el tiempo como una serie de acontecimientos que se dan de manera lineal; un conjunto de sucesos que llevan un orden del pasado hacia el futuro pasando por el presente. En términos generales la figura con la que lo representamos (y se acentuó mucho más gracias a la corriente positivista) es una línea horizontal en la que, de izquierda a derecha, el pasado se encuentra al inicio, en medio el presente y al final (infinito) de la línea el futuro. Ahora bien, las culturas prehispánicas mesoamericanas tuvieron una concepción distinta, mucho más rica y compleja que la que hemos adoptado y forma ya parte de nuestra cultura occidental.

En primer lugar, recordemos que la vida de las culturas mesoamericanas estaba permeada en todas sus esferas (sociales, culturales, económicas, bélicas, políticas, epistémicas, etcétera) por su religión. ${ }^{1}$ Para poder analizar cada esfera es necesario comprender su construcción teológica-epistémica; sin embargo, como hemos anotado, sólo analizaremos la categoría tiempo, misma que se encuentra inherentemente ligada a dicha construcción.

En el apartado "Las bases del conocimiento" de su libro El pasado indígena, López Austin anota que "Los tiempos [...] eran dioses que se extendían sobre el mundo, lo invadían y lo transformaban para abandonarlo después de haber impreso su huella" (224). Esta concepción es ya por sí misma sugerente, en virtud de que el tiempo es una entidad, y por lo mismo tiene una manera de actuar; existencia propia. El tiempo no es una intuición pura a priori, como en Kant, es decir, no es el hombre (en tanto humano) el que como sujeto trascendental (esto es, sujeto que es condición de posibilidad de conocimiento) otorga a los objetos sus cualidades; es el tiempo el que condiciona al sujeto, es el que decide sobre el humano. López Austin asegura que los tiempos eran dioses, es decir, el tiempo es intercambiable, coderivable con Dios, ya que Dios es tiempo y número y animal y objeto.

Vayamos por pasos.

\footnotetext{
${ }^{1}$ Entendemos aquí religión por el constructo en el que se vinculan la cosmogonía y la cosmología de las culturas mesoamericanas. Cabe resaltar que en dichas culturas el vínculo fundamental no es el territorio en el que se desarrollaron, sino las características que los unen: sus sistemas calendáricos, su sedentarismo, su politeísmo y la arquitectura.
} 
La religión mesoamericana se distinguió por elementos nucleares de gran persistencia que se articularon en torno a dos características básicas; fue una religión estrechamente vinculada a la agricultura [...] [y] también estuvo obsesivamente ligada al devenir del tiempo. Ambas obsesiones se fundieron en un solo símbolo: los dioses de la lluvia ("La religión, la magia y...", 242).

Hay dos calendarios en las culturas mesoamericanas que se asemejan cualitativa y cuantitativamente: los mexicas y los mayas. En los mexicas el calendario con funciones adivinatorias se llamaba tonalpohualli y los que hacían referencia a fiestas y épocas para sembrar recibía el nombre de xihuitl o año civil. ${ }^{2}$ El primero consta de 260 días y está conformado por la combinación de 20 días "nombrados" y 13 días "numerados". Su función, como se dijo, es adivinatoria, es decir, al nacer cada individuo está bajo la orden de un dios: el dios número y el dios nombre de la fecha de su nacimiento, y ambos influyen en el "destino" de dicha persona: "Cada unidad temporal era un destino. No era destino como hecho necesario y fatal, sino como una fuerza divina con características peculiares. Cada dios-día tenía diferente talante, determinados poderes y singulares apetencias que afectaban a los hombres" (López Austin, Dioses del..., 89), por ello, el hombre lleva impreso de manera inherente el calendario, es decir, el tiempo, esto es, un dios. Por otro lado, León-Portilla asegura que en los mayas: "todos los momentos de tiempo son llegadas y presencias de rostros de dioses que se aúnan y apartan sucesivamente, dejando sentir sus influencias y determinando sin cesar vida y muerte en el universo. Cada momento no es sólo presencia de un dios, sino suma de muchas presencias" (Tiempo y realidad..., 59).

Por su parte, el "calendario civil”" es el que posiblemente se acerca más a nuestra comprensión, ya que está compuesto por 18 periodos de 20 días cada uno: 360 días y cinco sobrantes que eran considerados "inútiles" o "perjudiciales". ${ }^{3}$ En los mayas tenemos dos calendarios semejantes: el primero (de 260 días) se llama "tzolkin, formado por la combinación de 13 numerales con 20 signos, y el calendario solar (haab), integrado

${ }^{2}$ Cfr. Hanns J. Prem, Manual de la antigua cronología mexicana.

${ }^{3}$ Cfr. Hanns J. Prem, Manual de la antigua cronología mexicana. 
por 18 meses de 20 días cada uno más cinco días aciagos, los uayeb, que complementan el ciclo anual del sol" (Valverde, Los mayas, 64).

Ambos calendarios (tonalpohualli-tzolkin, xihuitl-haab) fueron entregados por los dioses a los humanos; fueron ellos quienes les dieron este conocimiento para que supieran cómo conducirse en el mundo; es decir, todo objeto natural y animal es un dios, asimismo toda construcción humana también lo es: objetos, números, los calendarios, etcétera, estarán permeados por los dioses en virtud de que fueron ellos quienes enseñaron a los humanos cómo diseñarlos, y en un orden lógico fueron anteriores a la creación de la humanidad.

Hemos expuesto que los mitos mexicas y mayas indican que los dioses otorgaron las herramientas necesarias a la humanidad para poder conducirse en el mundo, lo que en orden lógico quiere decir que los dioses tenían una existencia previa a la humanidad; no obstante ese orden no sólo es lógico, sino también temporal: hubo un "entonces" previo a la existencia humana:

Los dioses pertenecen al otro tiempo y al otro espacio, antecedentes y causas del tiempo y del espacio mundanos. Están en el allá-entonces, pero también en el aquí-ahora ya que forman parte de todas las criaturas; están dentro de cada ser que se encuentre sobre la superficie de la tierra, en el espacio de los meteoros y en los cielos de los astros (López Austin, Dioses del..., 47-48).

Condición de posibilidad del tiempo y espacio mundanos es la existencia del otro tiempo y espacio: el de los dioses: "Sol, día y tiempo [mayas] no son entidades abstractas, sino realidad inmersa en el mundo de los mitos, aspectos de la deidad, origen de los ciclos que gobiernan todo lo que existe" (León-Portilla, Tiempo y realidad... 45). Nos detendremos brevemente aquí para hacer un contraste con la concepción espacio temporal que hemos heredado de un filósofo moderno fundamental: Kant. Para el filósofo de la Crítica de la razón pura, el individuo es un sujeto trascendental, es decir, un sujeto que es condición de posibilidad de conocimiento: sin sujeto no hay conocimiento, ya que es él quien con sus intuiciones puras a priori (tiempo-espacio) puede ordenar y percibir cualquier fenómeno. No son los objetos, no es la 
naturaleza los que imponen sus condiciones (como se asumía antes de la propuesta kantiana), no es la naturaleza la que nos condiciona. Mediante un giro copernicano, Kant otorga al sujeto la condición de posibilidad de conocimiento y no a un agente externo, agente que debe ser abordado por nosotros y no viceversa. En un primer momento, con las intuiciones puras a priori, ordenamos y percibimos, después, a partir de la facultad del entendimiento, espacio y tiempo son determinados bajo conceptos y categorías. ${ }^{4}$

Ahora bien, al colocar a la razón como objeto de análisis, Kant busca establecer los límites de la misma, para saber a qué tipo de conocimiento puede tener acceso, es decir, sus condiciones son epistémicas. Sin embargo, después de este primer momento, Kant nos lleva a un segundo estadio en el que ya no se cancela, se funda. Los límites de la razón se imponen de manera negativa, sin embargo, al saber qué objetos no podemos conocer, se construye una plataforma ontológica en la que nuestros objetos de conocimiento tienen existencia, y consecuentemente pueden ser sometidos a un análisis. Teniendo claro que Kant asume una posición epistémica y a su vez ontológica que repercutirá en la filosofía moderna y la posterior, ${ }^{5}$ así como en la vida cotidiana, haremos un contraste que nos parece vertebral para poder elaborar una crítica a dicha forma de asumir el mundo.

${ }^{4}$ Cfr. Kant, Crítica de la razón pura.

${ }^{5}$ De manera más específica, en el positivismo. Como sabemos, el capitalismo y el neoliberalismo han llevado a un extremo delirante la sistematización, predictibilidad, y manipulación de la técnica para dominar la naturaleza. Para inicios del siglo xx, con el avance agigantado de los medios de producción y tecnológicos, se pensaba que se vivía la "mejor de las modernidades", que se encontraban en el auge de la humanidad. No obstante, el comienzo de la Primera Guerra Mundial y su secuela, dieron un aviso al mundo capitalista: el progreso por el progreso mismo, el progreso como fin y como medio causaba estragos catastróficos, mismos que olvidamos entre otras cosas a causa de la idea del tiempo lineal. En tanto que el "pasado ya pasó" y no se vincula más con nosotros, no lo aprehendemos, no lo hacemos nuestro, no nos responsabilizamos de él. La obsesión con la que se avanza en la técnica para superar el desconocimiento que se tiene de lo externo, de lo otro, de aquello que no es humano, y a su vez, esa distancia de superioridad que se tiene con ello, ha derivado en la explotación irracional de recursos naturales así como su contaminación. No hay que olvidar el grave caso de la comunidad de Cochabamba en Bolivia, cuando en el año 2000 la Bechtell Corp. de San Francisco obtuvo el control total del agua de dicha comunidad, incluso la de la lluvia; y prohibió a sus habitantes la acumulación de agua de lluvia. 
En la comprensión mexica y maya, los dioses (entre otras cosas, desdoblados en entidades naturales) son la condición de posibilidad no solo del conocimiento, sino de la propia existencia de los humanos. Son ellos quienes deciden cómo van a conocer y qué conocerán los humanos; los que proporcionan las herramientas para que el individuo pueda conocer, y son a su vez quienes se sacrifican para que el mundo terrenal se objetive: dando lugar en su devenir a todo lo existente y a la humanidad misma: "Una característica de la cosmovisión maya es la unidad entre tiempo y espacio. El cosmos tuvo su origen cuando nació el sol y comenzó a moverse, y este movimiento creó a su vez un orden en la sucesión del tiempo y un orden en la configuración del espacio" (Florescano, Tiempo, espacio y memoria ..., 20) Sin dioses no hay conocimiento, ni ser; es por eso que los mexicas les deben la existencia y permanencia en el mundo. Esta es una de las razones primordiales por las que los rituales de ofrenda son cantos, danzas, autoflagelaciones o sacrificios humanos, que son vitales para seguir en el mundo:

al plasmarse en el calendario astronómico [maya] cada uno de los movimientos y pasos del sol, y al unirse de modo inextricable el calendario de los ritos y ceremonias religiosas con las actividades humanas, el orden social se convirtió en un reflejo del orden natural, en un ámbito que al reproducir con fidelidad los ejes del orden cósmico, buscaba infundirle similar regularidad y permanencia a las creaciones humanas (20).

Habíamos dicho ya que hubo un "entonces" en otro tiempo y espacio. Es importante resaltar que no es un "antes" o un "después"; es un "entonces". Analicemos esta categoría. Al hablar de un "antes" y un "después" nos referimos a un tiempo lineal en el que los eventos acontecen en un orden y pueden ser divididos: una tira que puede fraccionarse con el fin de estudiar cada evento inscrito en ella de manera individual. No obstante, el "allá-entonces" se manifiesta en el otro tiempo y espacio, por esta razón no corresponde a la temporalidad lineal; es un "allá-entonces" indefinido, imposible de ubicar y datar, ya que no tiene principio ni fin; es constante presente e inabarcable allá.

Una propuesta similar la encontramos en Walter Benjamin (18921940), filósofo fundamental para construir una crítica a la modernidad 
y posmodernidad. Sin conocer a fondo la comprensión del cosmos que tenían los mexicas y los mayas, Benjamin abraza esta idea: "El materialista histórico no puede renunciar al concepto de un presente que no es tránsito, en el cual el tiempo se equilibra y entra en un estado de detención" ("Tesis XVI", 53). El tiempo es un presente constante que permea toda actividad, es una yuxtaposición de tiempos individuales y cuando es necesario se logra una suspensión temporal colectiva. En los mexicas y mayas su aquí-ahora es, de nuevo, un ahora que no termina, un aquí que para ellos era el centro del mundo; un aquí que no comprendía solo el plano terrenal, sino los 13 cielos y los 9 estadios del inframundo: "como lo nota Thompson, sus sabios [mayas] 'concebían el tiempo como algo sin principio ni fin, lo que hacía posible proyectar cálculos acerca de momentos alejados en el pasado sin alcanzar jamás un punto de partida'" (León-Portilla, Tiempo y realidad..., 18-19). ${ }^{6}$ Aquí-ahora es un presente continuo en el que si bien se relatan acontecimientos de los hombres, es porque le antecede un deber con los dioses para seguir relatando su tiempo. El tiempo mexica y el maya no es un tiempo humano, es un tiempo de dioses, ya que son ellos quienes han enseñado a los hombres la numeración, los objetos, los animales, los calendarios que son ellos mismos. La concepción temporal mexica y maya es un presente constante que es la continuación del "allá-entonces" mítico. Si los calendarios, los números, toda construcción humana, si todo lo natural y el hombre es dios (al menos en un grado), entonces el tiempo sigue teniendo ese talante de suspensión: "Ambos tiempos-espacios pertenecen a los dioses. La diferencia entre ellos es que la sustancia encapsulante, la cáscara no puede cruzar los umbrales, y como las criaturas son los seres encapsulados, consideran su entorno como casa propia" (López Austin, Dioses del..., 68).

Sin embargo, surge la pregunta acerca de la aparente linealidad del tiempo mítico mexica y maya, la cual trataremos de salvarla aludiendo a dos elementos principales: lo cíclico del tiempo mítico y el permanente contacto que existe entre el tiempo mundano y el otro tiempo. Por un lado, León-Portilla expone que en los mayas:

${ }^{6}$ Cfr. Thompson, Maya Hieroglyphic Writing, 149. 
Las edades cósmicas que han existido, los "soles", son precisamente los días grandes de Kinh [...] Por obra de kinh todo está inmerso en la atmósfera de un tiempo que se hace presente y tiñe con la influencia de sus cargas los cuatro rumbos del mundo. Los rostros de los dioses-periodos sucesivamente se orientan por los grandes cuadrantes, determinando el destino y la vida de los humanos y de todo lo que existe. El espacio, y lo que en él hay, adquieren su verdadero sentido por obra de los ciclos de kihn (Tiempo y realidad..., 98). ${ }^{7}$

Por otro lado, en los mexicas existen cuatro edades que se viven antes del nacimiento del quinto sol; si bien se cuentan cuatro eras en las que hubo ensayos para que la humanidad pudiera existir, ese tiempo permanece en perenne contacto con el "aquí-ahora" de los mexicas. Ese tiempo también les pertenece, lo hacen suyo, se responsabilizan de él porque a él también se deben y sigue incidiendo en su "aquí-ahora":

Esta concepción está presente en los testimonios arqueológicos y en los mitos de esos pueblos, donde se observa que la creación de cosmos fue seguida por el "comienzo del tiempo" y se dice que ambos acontecimientos primordiales son los ejes articuladores del orden cósmico y del transcurrir humano. Antes de esta creación dominaba el caos. A partir de la creación del cosmos y del comienzo del tiempo el universo adquiere un orden, sus diversos componentes funcionan en armonía, y la sucesión de los días, las estaciones y el movimiento del sol transcurren de manera ininterrumpida, animando la regeneración continua de la vida (Florescano, Tiempo, espacio y memoria..., 9).

El "aquí-ahora" se alimenta del "allá-entonces", y el "allá entonces" sigue decidiendo sobre el "aquí-ahora"; es pertenencia constante, inacabable:

Dos clases de tiempo, dos clases de espacios establecieron firmemente sus linderos y se comunicaron por la apertura de los umbrales y el flujo de los ciclos. En un lado quedaron los dioses en su perenne convulsión

${ }^{7}$ Cabe mencionar que para León-Portilla la dupla tiempo-espacio es fundamental en el pensamiento maya. La comprensión del cosmos que ellos tienen está permeada de la duplicidad, es decir, todo tiene un complemento, una pareja que al estar en constante tensión da sentido al devenir. 
creadora, en su eterno presente, en la aventura que nunca llega a concluirse y el relato que no termina de narrarse. El otro fue el lado de los dioses capturados en la cáscara dura, pesada, visible, olorosa, donde el tiempo marcha en los pasos diferentes del pasado, el presente y el futuro, donde se torna y se retorna (López Austin, Dioses del..., 68).

Contrario a la tradición mexica y maya, el tiempo cotidiano en occidente se vive como una línea "vacía" que se llena con acciones, no obstante, esas acciones se olvidan y se rescatan sólo las "importantes"; las que tienen relevancia para la historia hegemónica: "La historia universal carece de un armazón teórico. Su procedimiento es aditivo: suministra la masa de hechos que se necesita para llenar un tiempo homogéneo" (Walter Benjamin, "Tesis XVII", 54).

La crítica que establece Walter Benjamin frente al historicismo y su tradición (entiéndase modernidad-capitalismo) es contundente, aguda, incisiva. Si tomamos la concepción mexica y maya del tiempo, podemos a su vez hacer una crítica a la manera como asumimos nuestra cotidianidad, permeada de una ideología neoliberal y capitalista. Dichos sistemas ideológicos-económicos dominan en su mayoría países occidentales, que procuraron un movimiento de "progreso" en el que vemos ese tiempo vacío y lineal que tiene que ser llenado a toda costa, sin importar su contenido. Vayamos un poco atrás. Ha habido un proceso para llegar a este tipo de sistema. Lukács nos explica que en el capitalismo moderno se encuentra con mucha mayor presencia la racionalización y la calculabilidad en la producción, que lleva a su vez, de manera inherente (¿necesaria?), las cualidades individuales del humano. Esta racionalidad tiene repercusiones en la subjetividad en la medida que hay un desgarramiento del sujeto. La manera en que el individuo participa en la división específica del trabajo hace que su actividad se reduzca a una función especializada, que por lo mismo, hace que su repetición sea mecánica. El proceso se vuelve calculable, pues su racionalización arrojó un tiempo de trabajo socialmente necesario. La calculabilidad es un eje en el análisis de Lukács en la medida en que describe los pasos que el individuo realiza, de manera que su conciencia se encuentra cosificada.

Nos dice Lukács que debe haber una "computabilidad" en el proceso del trabajo que nos lleve a una ruptura con la producción orgánica. Esto 
es, la manufactura de productos previos al capitalismo moderno estaba relacionada directamente con la naturaleza, con las necesidades de los individuos de la sociedad, y si los productos en ocasiones eran un excedente, no era su principal objetivo, ya que la producción era vista como parte de un todo orgánico en el que una necesidad era cubierta por otros y viceversa. En el momento en que la calculabilidad rige el proceso de producción, se genera una ruptura y el proceso ya no es orgánico, sino inorgánico, descompuesto, es decir especializado, y esto lleva al desgarramiento del sujeto. El desgarramiento tiene como consecuencia ver las particularidades del trabajador como anomalías en la medida en que son imprevisibles, y corren el peligro de romper con el proceso [icientificista?] universal, que requiere de la calculabilidad. El desgarramiento del sujeto desemboca en una actividad acrítica dentro de la maquinara capitalista, creando así un avance sin sentido, un progreso por la pura idea de progresar. El sujeto se desgarra, se ve ajeno en todas las esferas de las que él participa: política, económica, laboral, etcétera, dejando así al capitalismo la tarea de construirle un mundo en el que le haga parecer que todo se encuentra en orden y bienestar. ${ }^{8}$

Vemos que la concepción lineal del tiempo es inherente al sistema ideológico-económico en el que estamos inscritos; sin embargo, al comprender la temporalidad maya-mexica, tenemos la posibilidad de criticar esa linealidad y tratar de asumir el tiempo de otra manera. Si asumimos que el tiempo no es sólo una sucesión de acontecimientos, sino también pausa, en la medida en que podemos hacer puentes entre el pasado y el presente, el tiempo ya no sería un proceso que necesariamente iría hacia delante, no sería una máquina imparable y ajena a nosotros. Al reconocer una deuda con el pasado hay una suspensión en el presente, ya que es inimaginable mirar hacia un futuro mejor, cuando el pasado nos grita que hay momentos que no se han solucionado, que no han sido siquiera recordados. Abarcar la memoria que se encuentra ahí para traerla y reivindicarla. Asumir una crítica contra la idea de progreso que sigue permeando lo cotidiano; detenernos se ha vuelto indispensable:

${ }^{8}$ Cfr. Georg Lukács, Historia y conciencia de clase. 
[Benjamin] rescata la importancia de la interrupción del movimiento [...] La razón de todo esto es el progresismo a criticar, el cual cimentó con su fijación en el movimiento (por supuesto como uno predeterminado) toda posibilidad de una intervención activa por parte de los sujetos de la historia. Benjamin rescata esta posibilidad con la detención del movimiento (Gandler, “¿Por qué el ángel de la historia...”, 62).

Para el capitalismo el tiempo es forma, pura estructura que tiene la función de dominar; en cambio, para Benjamin el tiempo es contenido: movimiento vivo, contradictorio, que articulado bajo la acción de la memoria, se vuelve específico, una mónada que hace evidente lo que el sistema ha tratado de ocultar; la comprensión de la historia sería distinta, una historia que no busque un mejor futuro, infinito, que nunca llegará, sino que pueda suspenderse en su presente para tratar de reivindicar las faltas que hizo en el pasado:

El cronista que narra los acontecimientos, sin distinción entre los grandes y los pequeños, tiene en cuenta, al hacerlo, la siguiente verdad: de todo lo que sucedió alguna vez, nada debe considerarse perdido para la Historia. Es cierto: sólo a la humanidad redimida pertenece plenamente su pasado. Esto significa que sólo ella, en cada uno de sus momentos, puede citar su pasado. Cada uno de los instantes que ha vivido se convierte en una cita en la orden del día, y ese día es justamente el último (Benjamin, Tesis III, apud Löwy, Walter Benjamin..., 62).

Comprender la concepción mexica-maya del tiempo arroja la posibilidad de ampliar nuestra esfera epistémica, ética, política, en tanto individuos, que si bien por ser parte de una cultura occidental no podemos asumir una manera de ser con todas las implicaciones prehispánicas, sí podemos asumir una constante crítica a la manera en que nuestro propio devenir acontece, y en tanto individuos de una sociedad, la misma crítica puede ser llevada a ese "huracán", a esa "locomotora" cuasi imparable que es el progreso. La pausa, la suspensión, el freno se han vuelto indispensables, si es que todavía buscamos hacer estallar la maquinaria en la que estamos inmersos. 


\section{REFERENCIAS}

Benjamin, Walter, "Tesis XVI", en Tesis sobre la historia y otros fragmentos, trad. Bolívar Echeverría, México, Itaca-Universidad Autónoma de la Ciudad de México, 2008.

ECHEverríA, Bolívar (comp.), La mirada del ángel, México, Universidad Nacional Autónoma de México-Era, 2010.

Florescano, Enrique, Tiempo, espacio y memoria histórica entre los mayas, Chiapas, Gobierno del Estado de Chiapas, 1992.

GANDler, Stephan, “¿Por qué el ángel de la historia mira hacia atrás?”, en La mirada del ángel, Bolívar Echeverría (comp.), México, Universidad Nacional Autónoma de México-Era, 2010.

Kant, Emmanuel, Crítica de la razón pura, pról., trad., notas e índices Pedro Ribas, México, Taurus, 2009.

León-Portilla, Miguel, Tiempo y realidad en el pensamiento maya, México, Universidad Nacional Autónoma de México, 1986.

López Austin, Alfredo, "La religión, la magia y la cosmovisión", en Historia antigua de México, vol. IV: Aspectos fundamentales de la tradición cultural mesoamericana, México, Instituto Nacional de Antropología e Historia-Universidad Nacional Autónoma de México, 2001.

López Austin, Alfredo, Dioses del norte, Dioses del sur, México, Era, 2008.

López Austin, Alfredo, López Luján, Leonardo, El pasado indígena, México, Fondo de Cultura Económica, 2012.

Löwy, Michael, Walter Benjamin. Aviso de incendio, México, Fondo de Cultura Económica, 2003

LukÁcs, Georg, Historia y conciencia de clase, México, Grijalbo, 1969.

Prem, Hanns J., Manual de la antigua cronología mexicana, México, Centro de Investigaciones y Estudios Superiores de Antropología Social-Miguel Ángel Porrúa, 2008.

Valverde, María del Carmen, Los mayas, México, Tercer Milenio, 2000. 